Optimización de la formación de Asistentes Odontológicos

Alicia Elena Iantosca y Teresa Adela Butler

Revista ES (en y sobre Educación Superior)

Vol.1, N¹-2 / Fecha de publicación: 27/12/2021

e-ISSN: 2718-6539

https://revistas.unlp.edu.ar/ES/index

IIES - Facultad de Odontología

DOI: https://doi.org/10.24215/27186539e019

\title{
Optimización de la formación de Asistentes Odontológicos
}

\author{
Optimizing dental assistant training
}

\section{Otimização da formação de assistentedentário}

\section{Odontóloga Alicia Elena lantosca}

Directora de la Tecnicatura Universitaria en Asistencia Odontológica FO -

UNLP

aliantosca@yahoo.com.ar

Dra. en Odontología Teresa Adela Butler Secretaria de Articulación Docencia Asistencia FO - UNLP dikybutler@yahoo.com.ar 


\section{Resumen}

En el escenario de la Educación Superior la formación técnico profesional se plantea la expansión universal de la tecnología, la ciencia y la cultura, lo cual invita a cuidar y apreciar nuestros valores, a responder a la competitividad y a los retos de calidad y excelencia. La práctica odontológica se halla en un mundo cambiante, el sector salud enfrenta un desafío a las problemáticas de salud oral de la población. Aquí reflexionamos sobre la formación de Asistentes Odontológicos, siendo central fortalecer las prácticas pre-profesionales supervisadas. Para el abordaje del problema planteado se consideran como etapas de intervención la preparación preliminar para la acción, la planificación de las prácticas pre-profesionales supervisadas y la ejecución de las prácticas pre-profesionales. En el desarrollo de las etapas se contemplan reuniones de coordinación y organización de actividades, selección de espacios físicos y de docentes tutores, distribución de los alumnos de la Tecnicatura en Asistencia Odontológica en ámbitos asistenciales de diferentes especialidades y/o complejidad. También involucra el seguimiento y la evaluación de las prácticas pre-profesionales supervisadas. Esta estrategia estará reflejada, en el futuro, por la calidad técnico-científica y humanística en el desempeño del Asistente Odontológico, acorde a las demandas y como integrante del equipo odontológico.

\section{Abstract}

In the higher education scenario, vocational technical training is proposed the universal expansion of technology, science and culture, which invites us to take care of and appreciate our values, to respond to competitiveness and to the challenges of quality and excellence. Dental practice is in a changing world, the 
health sector faces a challenge to the oral health problems of the population. Here we reflect on the training of Dental Assistants, being central to strengthen supervised pre-professional practices. For the address of the problem raised, preliminary preparation for action, planning of supervised pre-professional practices and the implementation of pre-professional practices are considered as intervention stages. The development of the stages includes, coordination and organization meetings of activities, selection of physical spaces and tutor teachers, distribution of students of the Technique in Dental Assistance in care areas of different specialties and / or complexity. It also involves monitoring and evaluating supervised pre-professional practices. This strategy will be reflected, in the future, by the technical-scientific and humanistic quality in the performance of the Dental Assistant, according to the demands and as a member of the dental team.

\section{Resumo}

No cenário do ensino superior, propõe-se a formação técnica profissional a expansão universal da tecnologia, da ciência e da cultura, que nos convida a cuidar e apreciar os nossos valores, a responder à competitividade e aos desafios da qualidade e excelência. A prática dentária está num mundo em mudança, o sector da saúde enfrenta um desafio aos problemas de saúde oral da população. Aqui refletimos sobrea formação de Assistentes Dentários, sendo centrais para fortalecer as práticas pré-profissionais supervisionadas. A preparação preliminar para a ação, o planeamento das práticas préprofissionais supervisionadas e a implementação de práticas pré-profissionais são consideradas como fases de intervenção para a resolução do problema levantado. O desenvolvimento das etapas inclui, coordenação e reuniões de 
organização de atividades, seleção de espaços físicos e professores tutores, distribuição de alunos da Técnica de Assistência Dentária em áreas de cuidados de diferentes especialidades e/ou complexidade. Envolve também a monitorização e avaliação de práticas pré-profissionais supervisionadas. Esta estratégia refletir-se-á, no futuro, pela qualidade técnico-científica e humanista no desempenho do Assistente Dentário, de acordo com as exigências e como membro da equipa dentária.

\section{Palabras clave}

Formación, optimización, prácticas supervisadas, asistentes odontológicos, seguimiento.

\section{Keywords}

Training, optimization, supervised practices, assistants dental, follow-up.

\section{Palavras chave}

Formação, otimização, práticas supervisionadas, assistentes dentários, acompanhamento.

\section{Caracterización}

Este proyecto de intervención se desarrolla dentro del ámbito de la carrera "Tecnicatura Universitaria en Asistencia Odontológica" de la Facultad de Odontología de la Universidad Nacional de La Plata cuyo lugar de trabajo está radicado en CEAS (Centro de Enseñanza y Atención de la Salud), sede central de carreras universitarias vinculadas con la formación de recursos humanos 
para la salud. Éste edificio, ex Sanatorio de la Carne, está ubicado en calle 12 y 161 de la localidad de Berisso y ocupa una superficie cubierta de 4.917,45 m2, distribuidos en seis plantas. La Facultad de Odontología en el primer y segundo piso realiza diversas prácticas académicas, de gestión y otras actividades de formación. Además en planta baja cuenta con consultorios externos para atención a la comunidad.

La "Tecnicatura Universitaria en Asistencia Odontológica" es una creación que se suma a los esfuerzos que viene realizando la Universidad Nacional de La Plata en forma conjunta para la generación de nuevas carreras dando respuestas a las necesidades en salud de la comunidad. La misma asume el modelo de dicha universidad como "bien público y social como derecho humano universal y una responsabilidad del Estado" (Tauber, F., 2018).

El dictado se desarrolla en seis cuatrimestres a lo largo de 3 años con duración cuatrimestral y obligatoria otorgando el título de "Asistente Odontológico" que le permite al egresado trabajar como auxiliar del profesional odontólogo, en ámbitos públicos y privados. En el transcurso del ciclo lectivo la Tecnicatura desarrolla actividades, interrelacionadas y orientadas a obtener un resultado en concordancia con la misión. Utiliza el capital humano y los recursos materiales para producir en el cursante, durante el proceso formativo cambios adecuados que le otorgan la adquisición de nuevas competencias.

\section{Justificación}

Este proyecto de intervención corresponde a la línea de Educación Superior y Salud. En la Tecnicatura Universitaria Asistencia Odontológica los alumnos asisten a clases de modalidad teórico-práctica, de taller y de práctica asistencial en clínica que corresponden a los diversos espacios curriculares. 
Algunas de las prácticas se llevan a cabo en las diferentes clínicas de atención de la Facultad de Odontología de La Plata, donde los alumnos de asistencia odontológica forman parte del equipo asistiendo al operador, y otras prácticas las realizan en el edificio CEAS de la localidad de Berisso.

La práctica odontológica se halla en un mundo cambiante es decir, en los últimos años, han surgido, nuevas tecnologías, procedimientos y especialidades que hacen que el trabajo del profesional odontólogo sea muy variado. Así mismo, el sector salud enfrenta un gran desafío a las problemáticas de salud oral en la población.

Los modos de vinculación de la Universidad con el mundo del trabajo, con las necesidades de la sociedad y con los perfiles profesionales deseados son temas que sustentan la formación del recurso humano en la actualidad. En este sentido el proceso formativo en asistencia odontológica aporta claves para el constructivismo, principios y herramientas metodológicas para una práctica profesional con la calidad y el compromiso que se requiere.

Este contexto nos invita a pensar que el equipo de atención odontológica, individual o comunitaria, requiere de la participación del Técnico Universitario en Asistencia Odontológica como integrante del mismo. La complejidad de su participación y la responsabilidad que enfrenta en sus actividades exige espacios de formación de prácticas "in situ", modalidad que las alumnas de la Tecnicatura desarrollan ampliamente en su recorrido, acompañadas por un docente-tutor.

La OPS (Organización Panamericana de la Salud) destaca la importancia del perfil de los profesionales técnicos de los servicios de salud y la necesidad de enriquecimiento de los procesos formativos. 
Lo expresado motiva a reflexionar, respecto a la optimización en la formación de recursos humanos, que es fundamental fortalecerlas prácticas preprofesionales supervisadas, correspondiente al último año de la carreara, que comprenden un conjunto de tareas asistenciales de manera integral con conciencia ética y solidaria en espacios de atención odontológica de diferentes niveles y/o especialidades.

En este marco de ideas sería conveniente sumar a las prácticas asistenciales que se desarrollan en la Facultad de Odontología de la UNLP y en CEAS de Berisso, en consecuencia, interaccionar con los diferentes centros operativos de Odontología de Preventiva y Social de la UNLP para realizar algunas prácticas específicas donde también, aprender y brindar servicio contribuyen al fortalecimiento del proceso formativo. Expresa Tauber en Pensar en la Universidad "Una línea singular y compleja es la articulación de las carreras vinculadas a las ciencias de la salud entre sí y con las instituciones públicas privadas y municipales con distintos niveles de complejidad que funcionan en la región."

\section{Formación del asistente dental en el contexto actual de la Educación Superior en Argentina}

La formación de Asistentes dentales actualmente se encuentra en diferentes Facultades de Odontología nacionales y privadas como así también, en otras instituciones con distintos alcances. En las Universidades Nacionales de Cuyo y de La Plata la misma se desarrolla en el ámbito de la Facultades de Odontología como carrera de pregrado.

Las Instituciones de Educación Superior, en la etapa actual del país, están comprometidas con la formación de personal auxiliar de salud hacia un alto 
nivel y con la producción de conocimientos y adquisición de competencias frente a los nuevos desafíos sociales, económicos, técnicos y científicos. Las universidades referido a las tecnicaturas están participando en un proceso constante de superación académica, con una actitud dinámica, crítica, flexible, moderna, innovadora, generadora del saber, que propicia el cambio, que lo anticipa, que crece cualitativamente, de acuerdo a las transformaciones históricas, en una integración irreversible ciencia-sociedad- cultura.

En el escenario de la Educación Superior la formación técnico-profesional se plantea la expansión universal de la tecnología, la ciencia y la cultura, lo cual invita a cuidar y apreciar nuestros valores, así como también a responder a la competitividad y a los retos de calidad y excelencia.

La búsqueda de la superación y la excelencia académica es un imperativo ineludible para la Universidad Nacional de La Plata y es en esa dirección que se despliegan los esfuerzos.

La FOLP, hace algunos años, a través de un curso de dos años de duración dependiente de la Secretaría de Extensión y Planificación Universitaria, impulsó la formación de personal auxiliar para colaborar con el odontólogo en su práctica clínica y administrativa. A partir del año 2018 y ante la necesidad de dar respuesta de manera innovadora a un escenario cambiante, en profunda transformación y mejorar la enseñanza-aprendizaje y la producción de conocimientos abre la Tecnicatura Universitaria en Asistencia Odontológica considerando que de acuerdo con el artículo 28 inciso a) de la ley $N^{\circ} 24521$, una de las funciones básicas de la Universidad es la formación de profesionales, docentes y técnicos capaces de actuar con solidez profesional, espíritu crítico, sentido ético, atendiendo la demandas individuales, los requerimientos nacionales y regionales. De conformidad con lo dispuesto por 
los artículos 29, inciso e) y 42 de la mencionada Ley es facultad y responsabilidad atribuible a las instituciones Universitarias la creación de carreras y formulación y desarrollo de sus planes de estudio, así como la definición de sus conocimientos y capacidades que tales títulos certifican y las actividades para las que tienen competencia sus poseedores.

\section{Importancia de la formación de Asistentes Odontológicos en función de} las transformaciones de los servicios de salud

En los últimos años se evidencia una nueva etapa en el desarrollo y práctica de la odontología y las nuevas tendencias surgidas obligan a una rápida adaptación a ellas. Uno de los cambios más trascendentales, sin duda, se refiere a la conformación de los equipos odontológicos y el énfasis en el rol de cada integrante.

El equipo odontológico contemporáneo está formado por un grupo de técnicos y profesionales especializados en las distintas aristas de la odontología y la participación del Asistente Odontológico, continúa teniendo un rol protagónico muy importante a cuenta del significativo avance que su ámbito de acción ha experimentado durante las últimas décadas.

Desde hace algunos años, sus competencias específicas y genéricas se han ido expandiendo, es decir, paulatinamente, se han ido anexando a su desempeño roles muy diversos inherentes a tareas administrativas y de asistencia de la actividad del odontólogo.

Actualmente están ilustrados para, asegurar las condiciones sanitarias, de bioseguridad y ergonomía; realizar tareas de tipo administrativo, colaborar en 
actividades de promoción, prevención y procedimientos clínicos, asistir en la preparación de los equipos, de instrumental y de biomateriales utilizados en las diferentes prestaciones odontológicas.

Sosteniendo, entonces, que el equipo odontológico es un conjunto de técnicos y profesionales que se sistematizan de forma concreta para lograr un objetivo común, resulta incontrovertible aceptar que el aporte de la asistentes dentales influye decisivamente en el éxito y la calidad de la atención clínica por consiguiente, "la adaptación de los recursos y habilidades de una organización al entorno cambiante, aprovechando sus oportunidades y evaluando los riesgos en función de objetivos y metas..." (Tauber, F., 2008) debe tenerse en cuenta para la oportuna optimización de la formación del personal auxiliar que asiste a la comunidad odontológica.

El pensamiento estratégico indica "que frente a las variables que no podemos manejar (independientes) solo podemos posicionarnos, de modo que su probable evolución nos encuentre en la posición relativa favorable. Y frente a las variables sobre las que podamos incidir (dependientes) apliquemos los instrumentos más adecuados (eficaces) para manejarlas" (Garay 2003)

La orientación en la formación técnico profesional aproximada a la realidad de un mundo cambiante "de pronto no significa que plantear escenarios oriente las practicas lo que hace simplemente es poner a la luz a cerca de lo que se quiere hacer y hacia donde se quiere dirigir y obliga a hacerse cargo de eso que se sueña, de eso que se desea...." (Giordano, C., 2009)

La Tecnicatura en su recorrido que recién ha comenzado a transitar tiene la visión de ser reconocido como referente a partir de una formación integral que contemple las necesidades, la realidad social y no menos importante que se nutra con valores para que asuman la responsabilidad del trabajo en equipo en 
consecuencia asistir con calidez, calidad, dignidad, transparencia y en forma equitativa a la población que confía en la atención del servicio odontológico. Las universidades públicas, deben preparar a los estudiantes para ejercer como profesionales que se adecuen al contexto social, cultural y tecnológico en el que están insertos. Un contexto cada vez más complejo, en el que su permanencia sólo será posible si estos profesionales saben integrar adecuadamente la práctica profesional y el ejercicio de la responsabilidad social. Al respecto el artículo 4 de inciso a) de la Ley de Educación Superior no 24.521 señala como objetivo "Formar científicos, profesionales y técnicos, que se caractericen por la solidez de su formación y por su compromiso con la sociedad de la que forman parte".

Desde esta perspectiva, es fundamental enfatizar durante el proceso formativo un saber técnico con sustento ético-científico que permita el desempeño asistencial, en diferentes ámbitos laborales, con cierto grado de autonomía, compromiso y responsabilidad. A partir de su experiencia con la realidad en diferentes sectores de producción de bienes y servicios y mediante procesos de sistematización con autoreflexión crítica de saberes y de intervenciones, en el marco de la responsabilidad social universitaria, se profundiza la formación técnico-profesional-humanística.

\section{Reflexiones finales}

La formación de Asistentes Odontológicos en el marco universitario requiere la realización de prácticas y actividades asistenciales sensibles a los avances científicos-tecnológicos y a los dilemas económicos-sociales que atraviesa la comunidad. 
El fortalecimiento de las prácticas pre-profesionales supervisadas integrales, de manera crítica y reflexiva, representa una herramienta de formación significativa que permitirá a los estudiantes optimizar y enriquecer la propia práctica.

El desarrollo de prácticas en centros odontológicos de diferentes niveles de complejidad y/o especialidades, posibilitará al alumno adquirir el máximo grado de experiencia y de competencias, por consiguiente, mejorar la atención a la comunidad como auxiliares del profesional odontólogo.

La incorporación de los Asistentes Odontológicos en el mercado laboral de nuestro país, en este contexto, responderá a las demandas actuales, a la función social de la Universidad como institución pública y a los objetivos propuestos en este trabajo.

\section{Bibliografía}

Abate, S. M., \&Orellano, V. (2015). Notas sobre el curriculum universitario, prácticas profesionales y saberes en uso. Trayectorias universitarias, 1.

Arciniega, W. (2013). Rol de la o el asistente dental en odontología. Imbabura Ecuador.

Ardizzone, V. C. (Ed.). (2008). Manual práctico para el auxiliar de odontología. Elsevier España.

Buffa, S., Rinaudo, G., Díaz, I., Aybar, A., \& Carreras, R. (2017). La sistematización de prácticas como momento de reflexión y producción de conocimientos. Anuario de Investigaciones de la Facultad de Psicología, 3(1), 238-246. 
Campos, A. (2004). La contribución del asistente dental en el equipo odontológico del área metropolitana de Costa Rica. San José. Costa Rica: Universidad Latinoamericana de Ciencia y Tecnología.

Companioni, O. L. (2015). El Proceso de Formación Profesional desde un Punto de Vista Complejo e Histórico-Cultural. Actualidades Investigativas en Educación, 15(3), 567-589.

Cruz, V. (2015). Las prácticas pre-profesionales: un dispositivo de interpelación pedagógica. Trayectorias universitarias, 1.

De Alcázar, M. P. (2001). Las teorías clásicas de la comunicación: Balance de sus aportes y limitaciones a la luz del siglo XXI. Opción Año 2017: revista de Ciencias Humanas y Sociales, (36), 11-29.

Doberti, J .I (2018) La Organización Académica En La Universidad .EUDEBA Cap.2.

Durán, L. A. V. (2008). Formación: apuntes para su comprensión en la docencia universitaria. Profesorado. Revista de Currículum y Formación de Profesorado, 12(3), 1-14.

Ferry, G. (2004). Acerca del concepto de formación. Pedagogía de la formación, 53-73.

Fraile Lobato C. (2007) La Supervisión de la práctica profesional socioeducativa. Revista de Psicodidáctica. Universidad del País Vasco. España 12 (1).

Freire, P. (1998) Extensión o comunicación?: la concientización en el medio rural. Siglo XXI.

Garay, A (2003). Propuesta Metodológica para el Plan Estratégico de Mar del Plata y el Partido de General Pueyrredón, Mimeo de Concurso, Mar del 
Plata: Centro de Investigaciones Ambientales de la Facultad de Arquitectura de la Universidad Nacional de Mar del Plata.

García, M. M. B., \& Tirado, M. D. L. L. M. (2010). La sistematización de experiencias: producción de conocimientos desde y para la práctica. Revista Tendencias \& Retos, (15), 97-107.

Garza S. (1970). Utilización y adiestramiento del Personal Auxiliar. OPS/OMS. Washington.

Giordano, C. J. (2009). 23 tesis sobre la Tesis (Doctoral dissertation, Universidad Nacional de La Plata).

Honoré, B., \& Palacios, M. T. (1980). Para una teoría de la formación: dinámica de la formatividad. Madrid: Narcea.

Horruitiner Silva, P. (2006). La universidad cubana: el modelo de formación. La Habana: Editorial Félix Varela.

Jara Holliday, Ó. (2012). Sistematización de experiencias, investigación y evaluación: aproximaciones desde tres ángulos. F (x)= Educación Global Research, 1, 56-70.

Jara Holliday, Ó. (2018). La sistematización de experiencias: práctica y teoría para otros mundos posibles.

Ley de Educación Superior. Ley № 24.521 del Ministerio de Educación de la República Argentina.

Martínez, N. L., \& Colmenares, A. A. (2008). Exposición al óxido de etileno del personal auxiliar en odontología. Acta Odontológica Venezolana, 46(4).

Mattelart, A. (1976) La comunicación en el proceso de liberación. Siglo XXI. Buenos Aires.

Matus, C. (1987). Política, planificación y gobierno. Caracas: Fundación Altadir 
Ministerio de Educación. Buenos Aires. (2013) Orientaciones para el desarrollo de prácticas profesionales. Formación Técnica Superior. Documento de trabajo $N^{\circ} 1$.

Pérez de Maza, T. (2016). Sistematización de Experiencias en Contextos Universitarios. Guía Didáctica. Caracas, Venezuela: Ediciones del vicerrectorado académico.

Ramacciotti, B. (2015) .Contexto social concreto como escenario de articulación de una práctica profesional integradora. Trayectorias universitarias 1.

Sánchez, A. V., \&Leicea, O. V. (2007). El aprendizaje basado en competencias y el desarrollo de la dimensión social en las universidades. Educar. Barcelona. España. 40, 15-48.

Schôn, D. (1992). La formación de profesionales reflexivos. Paidós. Barcelona. España. Cap.1.

Tauber, F. (2008). La comunicación en la planificación y gestión para el desarrollo de las instituciones universitarias públicas argentinas: el caso de la Universidad Nacional de La Plata en el trienio junio 2004-mayo 2007 (Doctoral dissertation, Universidad Nacional de La Plata).

Tauber, F. (2018) Pensar En La Universidad. Proyecto institucional de la Universidad Nacional de La Plata (2018-2022).

Tommasino, H., \& Rodríguez, N. (2010). Tres tesis básicas sobre extensión y prácticas integrales en la Universidad de la República, bases y fundamentos. Publicado en Comisión Sectorial de Extensión y Actividades en el Medio, Integralidad, Tensiones y Perspectivas, Cuaderno de Extensión Nro, 1. 
Vallaeys, F. (2014). La responsabilidad social universitaria: un nuevo modelo universitario contra la mercantilización. Revista iberoamericana de educación superior, 5(12).

Villa, A., Poblete, M. (Dirs). 2007. Aprendizaje basado en competencias: una propuesta para la evaluación de las competencias genéricas ( $2^{\underline{a}}$ ed.). Bilbao: Mensajero 